\title{
An online monitoring, diagnosis and control system based on virtual instrument for CNC spindle
}

\author{
Pang Hong', Wu Xing ${ }^{2}$, Liu Tao ${ }^{3}$, Liu Chang ${ }^{4}$ \\ Faculty of Mechanical and Electrical Engineering, Kunming University of Science and Technology, \\ Kunming 650500, China \\ ${ }^{2}$ Corresponding author

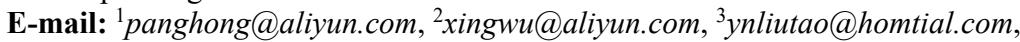 \\ 4liuchang3385@gmail.com
}

Received 19 September 2017; accepted 27 September 2017

DOI https://doi.org/10.21595/vp.2017.19168

Check for updates

Abstract. In the field of precision machining, the spindle is the "heart component" of the machining center. The dynamic performance of the spindle will directly affect the performance of the machine and the machining accuracy of the products. In order to avoid the above problems, an online monitoring, diagnosis and control system based on virtual instrument is designed for spindle. The system can monitor the operation condition of CNC electric spindle in real-time. Some classic signal processing and analysis methods are adopted such as time domain waveform, envelope spectrum and spectral kurtosis etc. The system is developed by LabVIEW language and on $107 \mathrm{Z}$ data acquisition system. The experiment platform for the system is a horizontal machining center of Dongyu CMV-1100A. The program is effective after preliminary test verification.

Keywords: electric spindle, online monitoring, virtual instrument, spectral kurtosis.

\section{Introduction}

Manufacturing industry is the national economy pillar industry of a country. Its technical level, equipment manufacturing capacity and the use proportion of high-end computer numerical control (CNC) machine tools is one of the important factors in measuring the national industrial level [1].

Promulgated by the State Council of the National Medium and Long Term Science and Technology Development Plan (2006-2020) [2] in 2006. The "major products and major facilities life prediction technology" as the focus of the development of China's foreword technology. The 12th Five-year development plan of machine tool industry [3] promulgated in 2011, the industry and information sector clearly said that the numerical control system and its function components is the key product development industry of China. Machining center performance indicators are accuracy, reliability and service life. Compared with similar foreign machine tools, the reliability of the domestic machine tools still existence a certain gap. And the important reason for lack of precision retention is the operational defects of the key functional components of the machine, such as machine tool spindle vibration, bearing failure, ball screw wear, thermal deformation and so on. And many of our heavy-duty processing center equipments are imported products, these key components once in the event of failure and defects, there will be a long maintenance cycle, the replacement parts are expensive, and affected the whole process. And thus, even have huge property losses and casualties. Also if maintenance or replace blindly will cause other damage, resulting in greater waste. Therefore, the online monitoring of key components for the processing center help to ensure the safe operation and improve the economic efficiency. "Large but not strong, much but not refined" is not the development of China's machine tool industry purpose, our goal is to make domestic machine tools stronger and more refined. The study is also based on the "Made in China 2025" [4] planning and pursuit the key technology and equipment to achieve a breakthrough, and achieve the theme of the manufacturing industry from big to strong.

Based on the development of traditional instrument technology, virtual instrument (VI) technology has also made great development in recent years. Providing a newer, faster and more efficient solution to industrial problem solving. It based on the PC, with a visual graphical display, 
data processing and peripheral interface programming environment, and technical staff can also design personalized graphical user interface according user needs.

In this paper, the signal processing technology and intelligent diagnostic technology of the machining center spindle failure, real-time online monitoring. Using VI programing LabVIEW to establish a set of online monitoring and diagnosis system. The system mainly uses the vibration and rotational speed of the spindle as the monitoring indicator. Signal processing technology using time domain, frequency domain and spectral kurtosis analysis method. The characteristic parameters that reflect the state of spindle are extracted from the collected data. The operation state of the current spindle is judged by the real-time online monitoring and data analysis. The alarm threshold of parameters is set through the benchmark data. Once an exception occurs, the system will automatically alarm, realizing the function of real-time monitoring of spindle state of machining center.

\section{General design of the system}

Taking into account the high precision and safety needs of CNC machining products, improvement measures are proposed from the monitoring and control of the process state. The experiment platform for the system is a horizontal machining center of Dongyu CMV-1100A. The system mainly includes the software and hardware. Based on the complexity of on-site environment, the monitoring points, wires arrangement, algorithms programming, and the interactivity with the $\mathrm{CNC}$ system. Data acquisition, signal process and analysis, real-time condition monitoring and control are deeply considered.

The hardware system structure includes machining center spindle module, sensor module, data acquisition module, data analysis module and computer module, as shown in Fig. 1.

The system will filter and amplification the original signal, and then analysis and diagnosis. Locate the fault location and generate a diagnostic log.

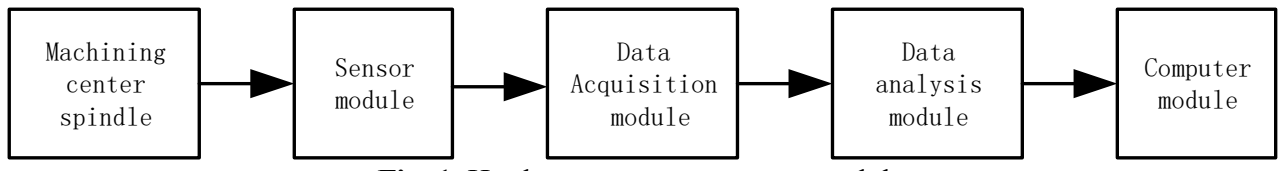

Fig. 1. Hardware system structure module

\subsection{Functional requirements of the system}

\subsubsection{Basic function requirement}

Physical quantities acquisition function: vibration, noise, many different kinds of sensors and the sensor sensitivity are needed.

Signal processing and analysis function: the filtering of the original signal, time domain analysis, frequency domain analysis and time-frequency domain analysis. The main methods used in this system are Fast Fourier Transform analysis, envelope spectrum, spectrum kurtosis etc.

Condition display function: shows the vibration value of the spindle machine process and estimates the time at which the failure may occur.

Fault diagnosis function: to diagnose the type, position and cause of fault.

Real-time condition monitoring function: according the result of fault diagnosis and signal analysis and monitor, to provide the current processing status, and the status light green is security, yellow is warning, red is alarm.

Diagnostic log generation function: generate txt documents based on diagnostics and maintenance, establish a health management file. 


\subsubsection{Special function requirements}

Reserved interface and channel, consumers can order the system functions according to their needs.

\subsection{Structure design of the system}

Sensors as the source of the signal, the monitoring point of CNC spindle is important. The selected data and preprocessed signals are transmitted to the industrial computer and displayed to the main interface of the system. Displays the current status based on the analysis results, and then according to the operation of the process to take the corresponding state of action. The system structure diagram is as shown in Fig. 2.

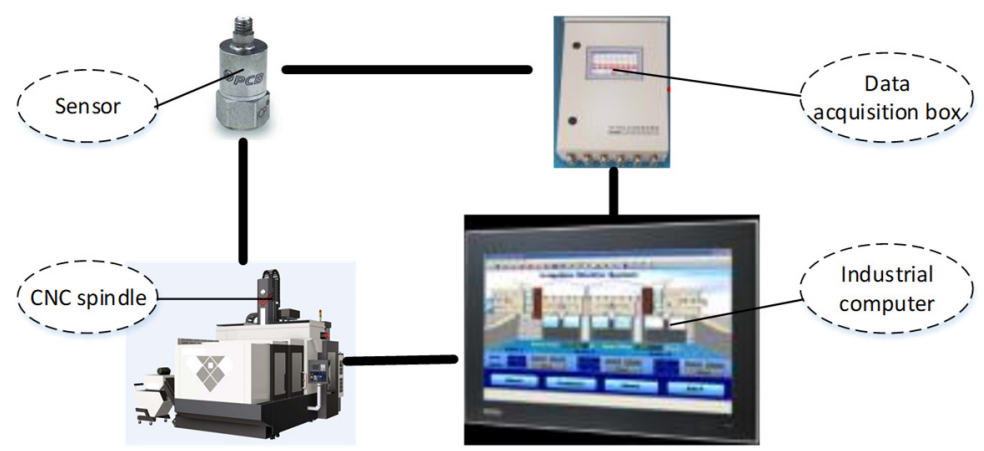

Fig. 2. Structure diagram of the system

\section{Functional modules of the system}

The system consists of different functional modules. Each module is distributed in the whole structure of the system, and namely data acquisition module, signal processing and analysis module, condition monitor module, fault diagnosis module and data management module. The structure of modules is as shown in Fig. 3.

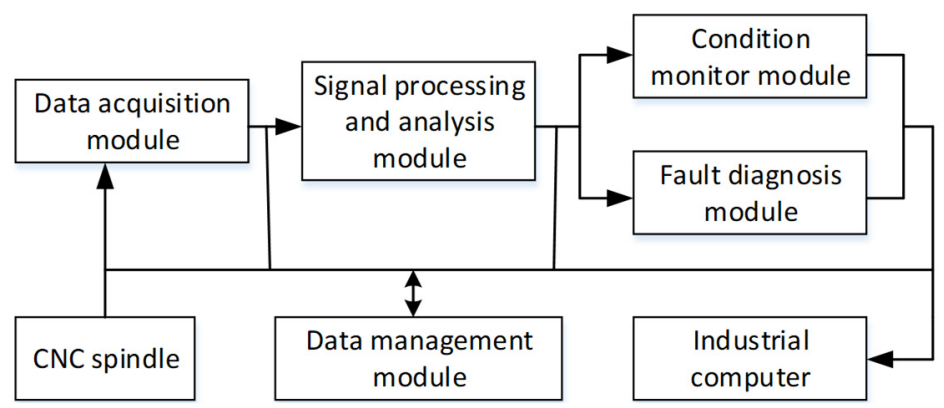

Fig. 3. The structure of modules

\subsection{The explanation of functional modules}

Data acquisition module can get real-time monitoring data through the sensors. The module also has some other parameter setting functions such as sampling rate adjustment, channel selection, sampling length control etc.

Signal processing and analysis module can process the original signal and extract the characteristic parameters by all kinds of algorithms and intelligent analysis means. This module's main function is processing and analysis the data in time domain, frequency domain and 
time-frequency domain. And the characteristic parameters include the amplitude of the vibration, peak-peak value, RMS, FFT, envelope spectrum analysis, wavelet packet analysis and spectral kurtosis analysis. And the spectral kurtosis analysis result as shown in Fig. 4.

The main function of the condition monitor module is to display the amplitude of the measuring point in real-time. If the state light is green, it means safe. If the state light is yellow, it means the value exceed the warning value and it's in an early warning state. If the state light is red, it means the value exceed the alarm value and it's in a dangerous alarm state. The system will then alert the operator to attention and arrangement of the maintenance. The condition monitor interface of the system is as shown in Fig. 5.

fb-kurt. $2-K_{\max }=4.6 @$ level 6, Bw= $400 \mathrm{~Hz}, f_{c}=5800 \mathrm{~Hz}$

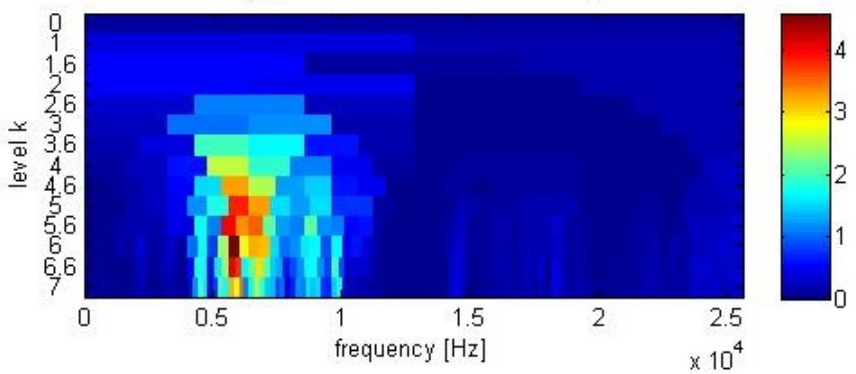

Fig. 4. Kurtosis analysis (1200 rpm)

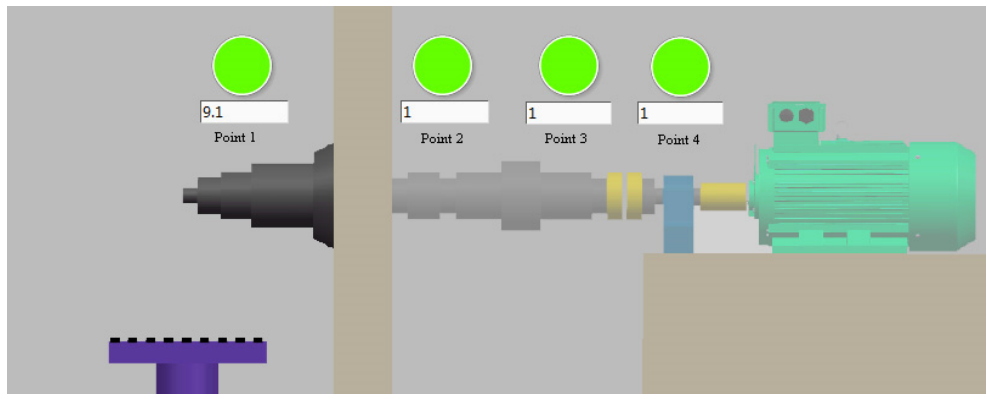

Fig. 5. The condition monitor interface

\section{Implementation of the system}

\subsection{Hardware design}

\subsubsection{Selection of sensors}

Five ICP vibration acceleration sensors, respectively mounted to spindle end and $X / Y / Z$ axis servo motor, sampling frequency $(0.5-10 \mathrm{KHz})$, manufactures $(\mathrm{PCB})$.

\subsubsection{Data acquisition box}

$107 Z$ data acquisition box is made in China. Support Modbus/TCP protocol, 16 channels vibration input and 16 channels current output.

\subsubsection{Other hardware}

Industrial Tablet PC by Taiwan Advantech. Core i5-4300U/4GB+2TB/15.6" TFT display. BNC junction box by PCB. Glass fiber material and 12 channels. 


\subsection{Software design}

The software system is developed with LabVIEWs. The software system includes the function modules mentioned in the third part above. At present, the system is being developed and completed the first vision, but the function modules need to be further perfected. The software main interface of the system is as shown in Fig. 6.

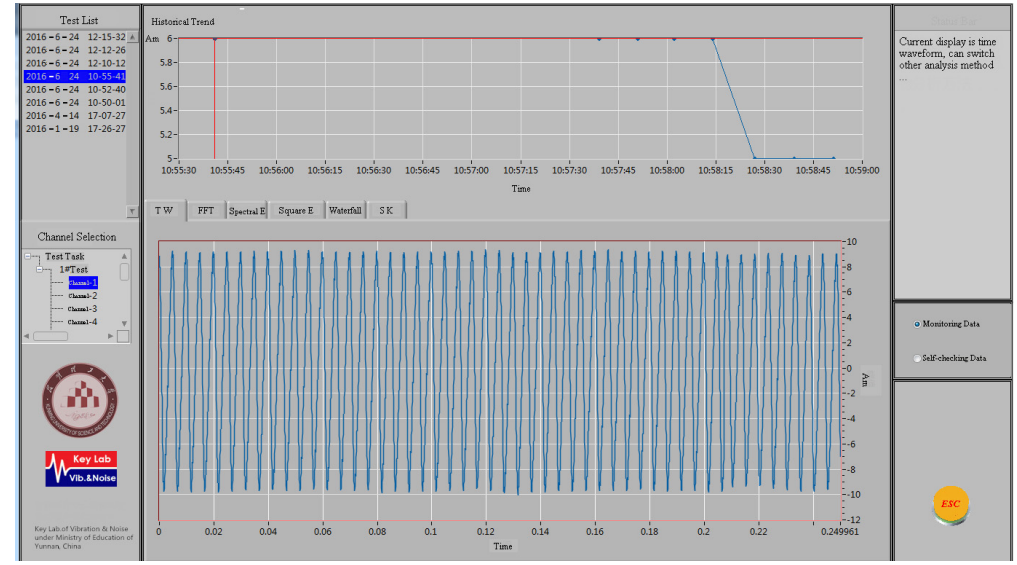

Fig. 6. The main interface

\section{Conclusions}

The study developed an online monitoring, diagnosis and control system for CNC machine spindle. The experiment platform for the system is a horizontal machining center of Dongyu CMV-1100A. The system is developed with LabVIEW language and on 107Z data acquisition system. The main functions of the system are: data acquisition, signal processing and analysis, condition monitor, fault diagnosis, data management and diagnostic log generation. The system can monitor the amplitude of the vibration. And even find the fault trend, locate the fault location and determine the type of failure. Achieving the condition assessment of the processing center equipment and reduce the cost of equipment maintenance. At present, the system is being developed and completed the first vision, but the function modules need to be further perfected.

\section{Acknowledgements}

Support from the National Science Foundation of China (Grant No. 51405211) is gratefully acknowledged. The thanks go to Xing Wu, Tao Liu, Chang Liu, Tao Wang, Liangfa Zheng, Cheng Tan, Bao Du and Kai Liang for support and work during the project. The authors also wish to thank Shanghai Huayang Measuring Instrument Co., Ltd. and Kunming Yunnei Power., Ltd. for their advices and help.

\section{References}

[1] Yang Zhaojun, Chen Chuanhai, Chen Fei, Li Guofa Progress in the research of reliability technology of machine tools. Journal of Mechanical Engineering, Vol. 49, Issue 20, 2013, p. 130-139.

[2] http://www.most.gov.cn/mostinfo/xinxifenlei/gjkjgh/200811/t20081129_65774.htm, 2006.

[3] http://www.docin.com/p-1264955005.html.

[4] Zhou Ji Intelligent manufacturing - main direction of "Made in China 2025". Mechanical Engineering, Vol. 26, Issue 17, 2015, p. 2273-2284.

[5] Vafaei S., Rahnejat H., Aini R. Vibration monitoring of high speed spindles using spectral analysis techniques. International Journal of Machine Tools and Manufacture, Vol. 42, Issue 11, 2002, p. 1223-1234. 
[6] Cao H., Zhang X., Chen X. The concept and progress of intelligent spindles: a review. International Journal of Machine Tools and Manufacture, Vol. 112, 2016, p. 21-52.

[7] Liu F., Zhu H., Shao X., et al. Analysis of horizontal machining center field failure data based on generalized linear mixed model - a case study. Quality and Reliability Engineering International, Vol. 27, Issue 2, 2015, p. 239-248.

[8] Chang C. F., Chen J. J. Vibration monitoring of motorized spindles using spectral analysis techniques. Mechatronics, Vol. 19, Issue 5, 2009, p. 726-734.

[9] Kegg R. L. One-line machine and process diagnostics. CIRP Annals, Vol. 33, Issue 2, 1984, p. 469-473.

[10] Zhou Dezhao, Zhang Jinming, Jiang Zhinong LabVIEW - Based data acquisition and processing of the rotor vibration signal. Instrumentation and Measurement, Vol. 24, Issue 3, 2005, p. 62-64.

[11] Sawalhi N., Randall R. B., Endo H. The enhancement of fault detection and diagnosis in rolling element bearings using minimum entropy deconvolution combined with spectral kurtosis. Mechanical Systems and Signal Processing, Vol. 21, Issue 6, 2007, p. 2616-2633.

[12] Antoni J., Randall R. B. The spectral kurtosis: application to the vibratory surveillance and diagnostics of rotating machines. Mechanical Systems and Signal Processing, Vol. 20, Issue 2, 2006, p. 308-331. 